\title{
Quantic Analysis of the Adherence of a Gram- Negative Bacteria in A HEPA Filter
}

\author{
Iliana Herrera-Cantú ${ }^{1}$, Karina García-Aguilar ${ }^{1,2}$, Erick Pedraza-Gress ${ }^{1}$, Emmanuel \\ Vázquez - López ${ }^{1}$, Juan Jesús García-Mar ${ }^{1}$, Lillhian Arely Flores-González ${ }^{1}$, Manuel \\ Aparicio -Razo ${ }^{1,4}$, Oscar Sánchez-Parada ${ }^{3}$ and Manuel González-Pérez ${ }^{1,5}$ \\ ${ }^{1}$ Universidad Popular Autónoma del Estado de Puebla A.C. (UPAEP). Centro \\ Interdisciplinario De Posgrados (CIP). Posgrado en Ciencias de la Ingeniería Biomédica. \\ ${ }^{2}$ Instituto Tecnológico Superior de Coatzacoalcos. Academia de Ingeniería Bioquímica \\ ${ }^{3}$ Escuela de Medicina Universidad Popular Autónoma del Estado de Puebla (UPAEP). \\ ${ }^{4}$ Benemérita Universidad Autónoma de Puebla (BUAP), Facultad de Ciencias de la Electrónica \\ ${ }^{5}$ Sistema Nacional De Investigadores. Nivel 1.
}

\begin{abstract}
It is known that Gram-negative bacteria $(G N B)$ are the most frequent bacteria in hospital units. It is also known that GNBs generate a greater number of nosocomial infections in critical areas. In the present work, the adhesion of the bacterial cell wall $(B C W)$ to the compounds of the material layers of a high efficiency filter (HEPA) was analyzed. The analysis was carried out by means of molecular simulation and quantum chemistry. The BCW and HEPA molecules were designed using Hyperchem software for simulation. The calculations of the quantum interactions of the molecules were carried out using the theory of the electron transfer coefficient (ETC). It obtained from 4 to 6 compounds that are more likely to interact even as a chemical reaction. The compounds of the glass fibers are the ones that work best for the adhesion and destruction of the $B C W$.
\end{abstract}

Keywords- Quantum study, Bandgap, Electron Transfer Coefficient, Adhesion, Bacterial Cell Wall, HEPA filter.

\section{INTRODUCTION}

In the hospital units, we can find an environment contaminated by the diversity of patients with their illnesses. Within this contaminated environment, there are the problem of the generation of acquired infections, called intra-hospital or nosocomial infection (NI) infections that aggravate the quality of the patient's health, generating extra costs of stay and treatment. In Mexico it has been estimated that the frequency of NI in hospital units varies from 2.1 to $15.8 \%$, the most common being pneumonia, urinary infection, surgical infection, and bacteremia [1]. More than 1.4 million people in the world get infections in the hospital. Between 5\% and $10 \%$ of patients who enter modern hospitals in the developed world will contract one or more infections [2].
Most frequent Gram-negative bacteria in a hospital Bacteria with rod characteristics (bacilli) are classified based on the structure of their cell wall in Gram-positive bacteria and Gram-negative bacteria.

The data provided by the U.S. National Healthcare Safety Network (NHSN) indicate that GNB is responsible for more than $30 \%$ of the NI and that these bacteria predominate in cases of in-hospital pneumonia (NIH) in $47 \%$, and urinary tract infections in $45 \%$. Infections caused by GNB trigger problematic characteristics due to their ability to adapt and acquire genes that code for the mechanisms of resistance to antibiotics [3].

Some GNB organisms that predominate over respiratory infections (causing NIH) are Haemophilus influenzae, Klebsiella pneumoniae, Legionella pneumophila, Pseudomonas aeruginosa. On urinary infections are: Escherichia coli, Proteus mirabilis, Enterobacter cloacae, Serratia marcescens; on blood infections are: Klebsiella, Escherichia coli, Enterobacter and Pseudomonas aeruginosa; and on gastrointestinal infections are: Helicobacter pylori, Salmonella enteritidis, Salmonella typhi [4].

\section{Bacterial cell wall}

The bacterial cell wall (CBW) has a thickness of $10 \times 25$ nanometers (nm) and is constituted by murein, lipoproteins, and lipopolysaccharides that constitute up to $80 \%$ of the weight of the cell wall. In Gram-positive bacteria $(\mathrm{Gram}+)$ the cell wall is formed by several layers of murein or peptidoglycan. In Gram-negative bacteria (Gram -) the wall is formed by a single layer of murein and by lipoproteins [5].

The ETC theory.

The BG is defined as the energy difference between the valence band and the conduction band. In the $\mathrm{BG}$ there 
are no electronic states available; this means that when an electric field is applied the electrons cannot increase their energy.

In quantum theory, it is known as HOMO and LUMO, and in the old theory they are known as E- and E +. The LUMO is defined as the range of electronic energy that allows acceleration in electrons by the presence of electrical currents and is also called conduction band; HOMO is defined as the highest energy interval that is occupied by electrons in absolute zero value and is called valence band. The HOMO is the most electron-filled orbital, while the LUMO is the orbital that lacks electrons. The HOMO equaled to zero (HOMO 0) is the last layer full of orbitals meaning that it is in the last valence orbital. The LUMO equaled to zero (LUMO 0) is the last layer that lacks electrons

EP is defined as the total potential energy of the molecule. It is an electrostatic field vector that is defined as the potential that the electron needs to jump the Bohr radius (0.53 Armstrong) by its calculated natural electromotive force (EMF). The negative E value (E-) is the electrostatic potential with negative poles, while the positive $E$ value $(\mathrm{E}+)$ is the proton-electron potential [6]. The EP, in other words, means that having $1 \mathrm{EP}$ is having 1 volt for Armstrong. The EP is obtained by the absolute difference of $\mathrm{E}-$ and $\mathrm{E}+$.

The ETC is defined as the dimensionless parameter that describes an electrochemical reaction, which is interpreted as the number of times the potential energy needs to jump to the BG. It is calculated by dividing the BG and the EP entirely. That is, if it has a BG of 10 and an ETC of 40, it means that you need 40 times the EP value in $\mathrm{EV}$ so that the $\mathrm{BG}$ of 10 jumps from the HOMO to LUMO.

The quantum well is defined as the area in which the value of the ETC may fall. These zones are divided into 3 (Fig. 1):

1. The hight probability area. It is the area below the inferior limit of the ETC of a compound (ZONE I) where a very high probability that a chemical reaction or simple molecular interaction occurs.

2. The medium probability area. It is the area between the inferior and superior limit of the ETCs of both compound interacting (ZONE II).

3. The low probability area. It is the zone above the superior limit of the ETC of a compound (ZONE III). It is the zone where a very low probability molecular interaction occurs [7 - 11].

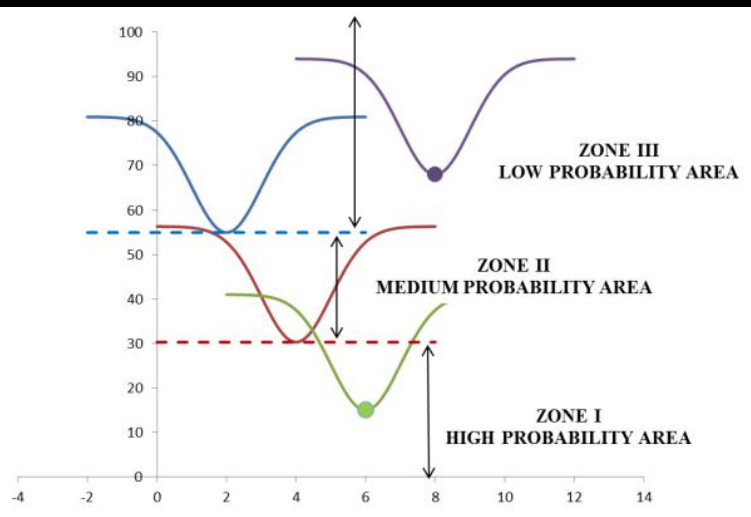

Fig.1: Probability zones for molecular interaction according to their quantum wells ETCs

\section{HEPA air filters}

The objective of high-efficiency air filters (HEPA) is to remove the particles that are inside an air conditioning system for the purification of the environment.These filters are composed of a random fiber mesh with diameters between 0.5 and 2.0 micrometers $(\mu \mathrm{m})$ composed of layers of various compounds such as cellulose, synthetic fibers (polyacrylonitrile) or glass fibers, also use latex and chitosan.

\section{MATERIALS AND METHODS}

We carried out the values, and simulation of the molecules of the compounds with the HYPERCHEM software. The software must be in SEMIEMPIRICAL form to perform the calculation of the $\mathrm{BG}$, the EP, and the ETC. When the complete molecule is drawn, the values of HOMO (-), LUMO (+), E- and E + are obtained, in value at zero and with a density of 0.015 . The values that are recorded will be captured in an Excel sheet, and the operations will be carried out to obtain BG, EP, ETC. To obtain the cross-band of the compounds, it is done taking the value HOMO and E- of the first compound and the value of the LUMO and E + the second compound. The lower ETC of the cross band will be the value that will determine which compound will be more reactive and will serve as data that will be placed in the quantum well graphs. To establish the limits of the graphs, the higher ETC will be placed at the upper limit and the lower ETC as, the lower limit of the compounds to be compared.

The molecules that were taken for the simulation are listed in Table 1.

Table.1: Molecules interacting

\begin{tabular}{|l|c|c|}
\hline Chemical substance & Abbreviation & Molecule \\
\hline Bacterial cell wall & & CBW \\
& & $\ldots$ \\
\hline
\end{tabular}

Molecules of HEPA filter compounds 


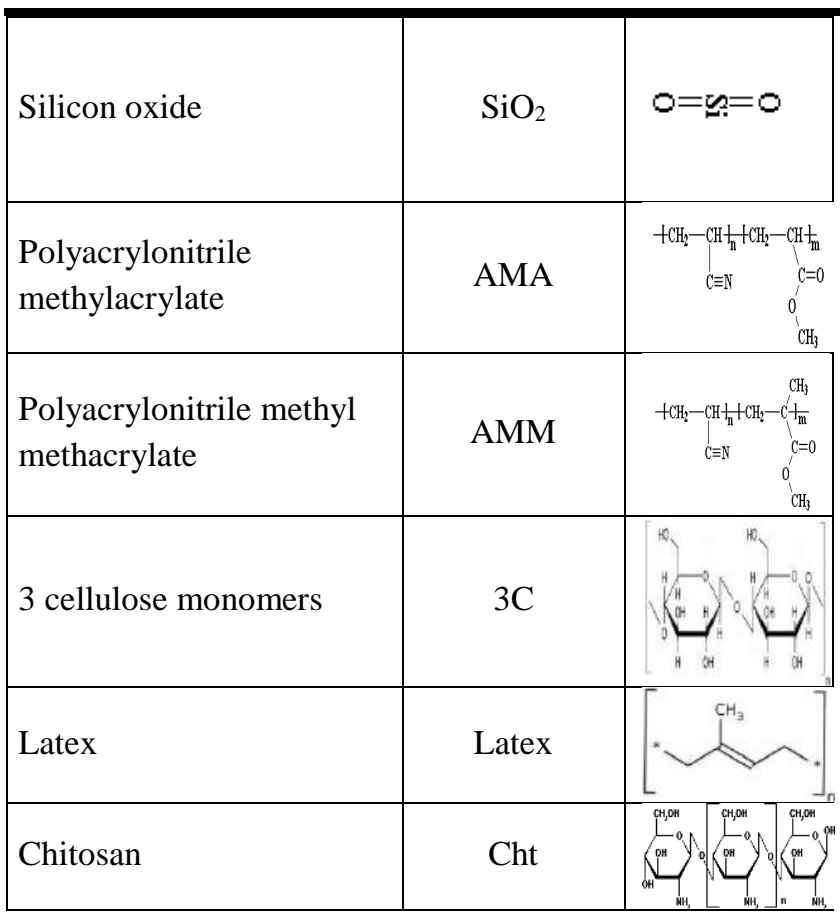

ETC

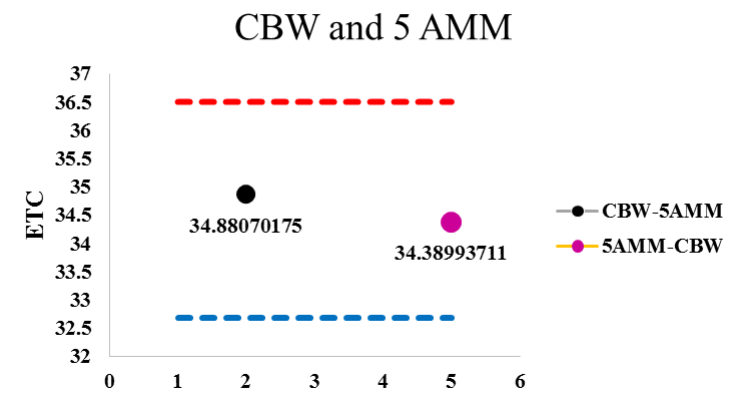

Fig.4: It can be seen that both cross-band options are in the medium probability zone; but, there is more affinity of the WCB as an oxidizing agent.

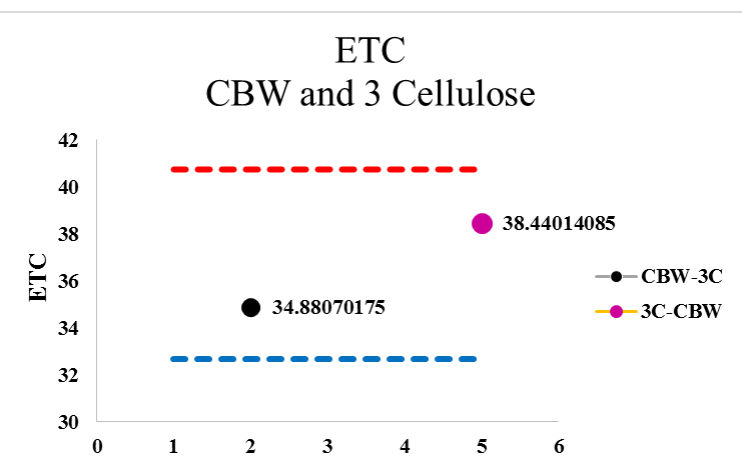

Fig.5: The CBW tends to behave as a reducing agent and adhere to the lower limit. Both cross-band interactions are in the medium probability zone.

In Fig 4 and Fig 6 it can be argued that by keeping both compounds in the middle range of the quantum well limit will depend on external conditions to know which compound is most likely to react as a reducing agent and achieve adherence.

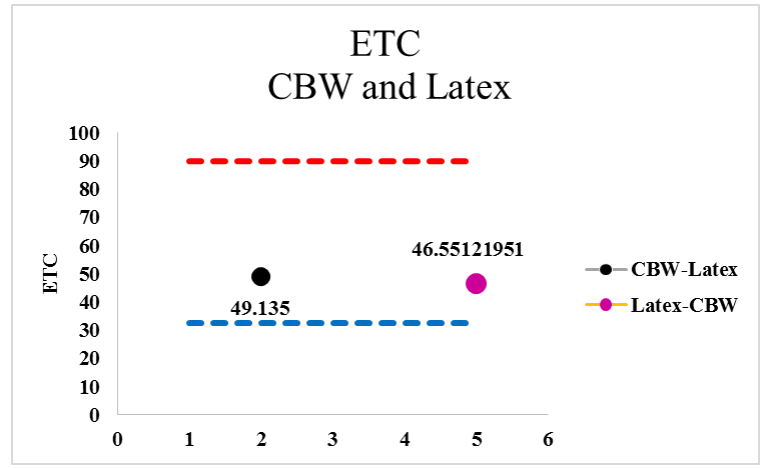

Fig.6: Both cross-band interactions are located in the medium probability zone. It is a similar case shown in Figure 4; but the difference is highlighted in the values of the ETCs.

In Fig 3 it can be argued that the value of the ETC obtained from the crossed band exceed the lower limit of the quantum well, meaning that the PCB acts as a reducing agent with a reaction probability above $75 \%$.

Fig.3: Both interactions of cross-bands are outside the medium probability zone; but, the $C B W$ (as a reducing agent) has a lower ETC than the lower limit that places it in the high probability zone. 


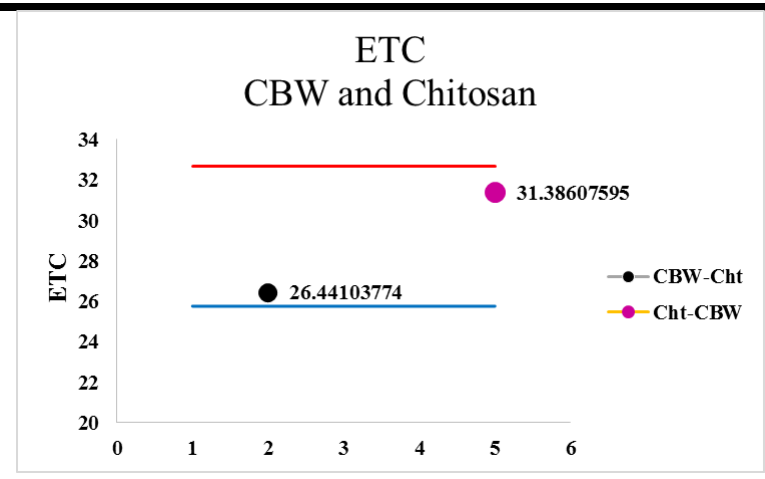

Fig.7: Both cross-band interactions are located in the medium probability zone. It is a similar case shown in Figure 2; but the difference is highlighted in the values of the ETCs.

It can be determined that the closer the value of the crossband ETC is to the lower limit (which represents the $75 \%$ reaction probability). It is determined that this compound will be the best compound to act as an oxidant since it is sought that the PCB is the one that acts as a reducing agent to achieve its adherence to the compounds. According to this, the compound that works best as an oxidant concerning $\mathrm{PCB}$ is silicon, for obtaining the smallest value of ETC in its cross-band.

\section{CONCLUSION}

The lower cross-band values of the ETC indicate that they are the best reactants. According to this lower value in the crossed bands of ETC, the compounds that work best as oxidants are Silicon (24,073), Chitosan (26,441), Polyacrylonitrile-Methyl acrylate $(29,383)$ and Cellulose $(35,150)$. PCB is more likely to react by adhering to the compound as long as it works as a reducer or antioxidant. Among the compounds used, the silicon yields a lower value of ETC (24,073), establishing that it is the material that will work best for the attraction of the PCB molecules. For the compounds with the same probability of reacting (latex and polyacrylonitrilemethylmethacrylate), it will depend on external conditions to know which is more likely to adhere. In conclusion, the filters made with higher layers of silicon or glass fibers will be the best for the adhesion of the $\mathrm{PCB}$, improving the environment of microbial agents.

\section{REFERENCES}

[1] Firmin H. Aikpo, Miriac Dimitri S. Ahouanse, Lucien Agbandji, Patrick A. Edorh, Christophe S. Houssou(2017).Assessment of contamination of soil by pesticides in Djidja's cotton area in Benin. International Journal of Advanced Engineering Research and Science (ISSN : 2349-6495(P) | 24561908(O)),4(7), 001-005.
[2] Salud, secretaría de. 2011. dirección general de evaluación del desempeño. [en línea] noviembre de 2011. medición de la prevalencia de infecciones nosocomiales en hospitales generales.

[3] Pittet, d., \& donalson, 1. (2006). una atención limpia es una atención segura. primera iniciativa mundial de la alianza mundial de la oms para la seguridad del paciente. ific, 2, 135-40.

[4] Horan, teresa c.; andrus, mary; dudeck, margaret a. cdc/nhsn surveillance definition of health careassociated infection and criteria for specific types of infections in the acute care setting. american journal of infection control, 2008, vol. 36, no 5, p. 309-332.

[5] Arias-flores, r., rosado-quiab, u., vargas-valerio, a., \& grajales-muñiz, c. (2016). microorganisms responsible of nosocomial infections in the mexican social security institute. revista médica del instituto mexicano del seguro social, 54(1), 20.

[6] Pírez, m.; mota, m. morfología y estructura bacteriana. revista en internet], 2000, vol. 3, no 2, p. 23-42.

[7] Mondragón-jiménez, jesús francisco, et al. interaction of metilendioximetanfetamina vs. neurotransmitters and the relationship by the quantum method. 2017.

[8] González-Pérez, M. (2017) chemical-quantum analysis of the aggressiveness of glucose and its appeasement with atp inside the cell. and water as an excellent antioxidant. World Journal of Pharmacy and Pharmaceutical Sciences. Volume 6, Issue 4, 9599. DOI: 10.20959/wjpps20174-8940.

[9] González-Pérez, M. (2017). Quantum Theory of the Electron Transfer Coefficient. International Journal of Advanced Engineering, Management and Science (IJAEMS).Vol-3, Issue-10, Oct- 2017. doi.org/10.24001/ijaems.

[10] González-Pérez, M. (2017). Quantum modeling to determine the carcinogenic potential of aflatoxin B1 produced by Aspegillus sp and its metabolic derivate aflatoxin M1. Mexican Journal of Biotechnology 2017, 2(2):255-270.

[11] Pacheco-García, PF; Perez-Gonzalez; Ramos-Flores A; Flores-Gonzalez, LA; Lopez-Oglesby, JM; Gonzalez-Perez, M. Experimental study and calculation of the electron transfer coefficients on the dissolution behavior of chitosan in organic acids. International Journal of Advanced Engineering, Management and Science (IJAEMS). Vol-3, Issue-6, Jun- 2017. doi.org/10.24001/ijaems. 